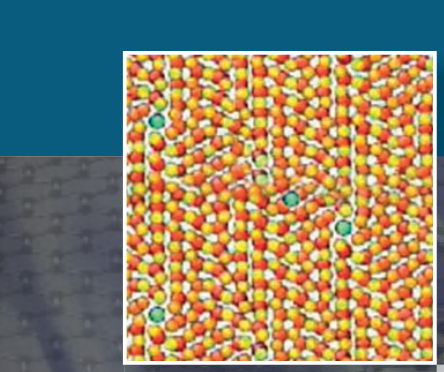

\title{
Mechanical behavior of nanocomposites
}

\author{
Markus J. Buehler and Amit Misra, Guest Editors \\ While initial interest in nanocomposites was to explore size effects in mechanical behavior, \\ current research is focused around the new paradigm of interface-dominated/enabled \\ mechanical behavior. The use of advanced computational tools to accelerate the discovery, \\ design, and fundamental understanding of behavior is highlighted along with novel synthesis \\ and in situ nanomechanical characterization tools. Designed interfaces can be used as building \\ blocks to create new forms of hierarchical composites with unprecedented mechanical \\ and physical behavior. New frontiers in the field of mechanical behavior of nanocomposites \\ involving metallic, nanocarbon, bioinspired, and biomaterials are reviewed.
}

\section{Introduction}

Nanocomposites are composed of two or more phases engineered to have nanoscale architectures with a high density of interfaces, and often with nested, hierarchical geometries. By engineering the length scales, morphology, crystal structure, and chemistry of interfaces, nanocomposites can deliver mechanical properties that exceed what could be achieved from a single-phase material alone. Metal nanolayered composites consisting of alternating layers of two different metals with nanoscale repeat layer spacing exhibit ultrahigh strengths that greatly exceed the strength from simple volume fraction estimation. ${ }^{1}$ Using severe plastic deformation, interfaceengineered nanocrystalline steel has been produced with a far-from-equilibrium structure containing supersaturated carbon at iron subgrain boundaries and a tensile strength of $7 \mathrm{GPa}$, which is perhaps the strongest bulk ductile metallic nanocomposite reported. ${ }^{2}$ Biological systems such as nacre, a composite consisting of aragonite platelets separated by a thin organic layer, are known to have superior toughness. ${ }^{3}$ Bioinspired hierarchical and nanolaminated multiphase steels have been developed that exhibit superior fatigue resistance at ultrahigh flow strengths. ${ }^{4}$

The articles in this issue of MRS Bulletin highlight recent developments in the mechanics of nanocomposites that are composed of a combination of metallic, polymer, biological, and ceramic materials. They also outline the challenges and opportunities for future research directions. Advances in computational and experimental approaches to guide the synthesis and elucidate novel and unusual aspects of nanomechanical behavior of composites are highlighted.

\section{Metal-based nanocomposite systems}

Earlier work on metallic nanocomposites focused on simple geometries such as laminates, which contain metals with cubic crystal symmetries to explore size effects in plasticity following the "smaller is stronger" paradigm,, 56 and later on, expanded to other material systems such as metal-ceramic ${ }^{7,8}$ and metal-metallic glass. ${ }^{9-11}$ Recently, interest in this field has broadened to explore new forms of metal-based nanocomposites such as metal/nanocarbon, cubic/noncubic, and designed three-dimensional (3D) morphologies. A new paradigm of interface-dominant or interface-enabled mechanical behavior ("it is all about interfaces") has emerged. ${ }^{12-16}$

Metallic nanolayered composites exhibit ultrahigh yield strengths, typically an order of magnitude higher than annealed bulk metals. This is due to interfacial confinement of dislocations and suppression of dislocation pileup-based behavior at the nanoscale. However, at the limit of a few nanometers, nanolaminates fail by shear bands initiated when the interfacial confinement breaks down and single dislocations, without the mechanical advantage of a pileup, transmit shear across the interphase boundaries. ${ }^{17}$ Suppression of shear bands in nanocomposites while taking advantage of the unprecedented high strengths enabled by nanostructuring has been a 


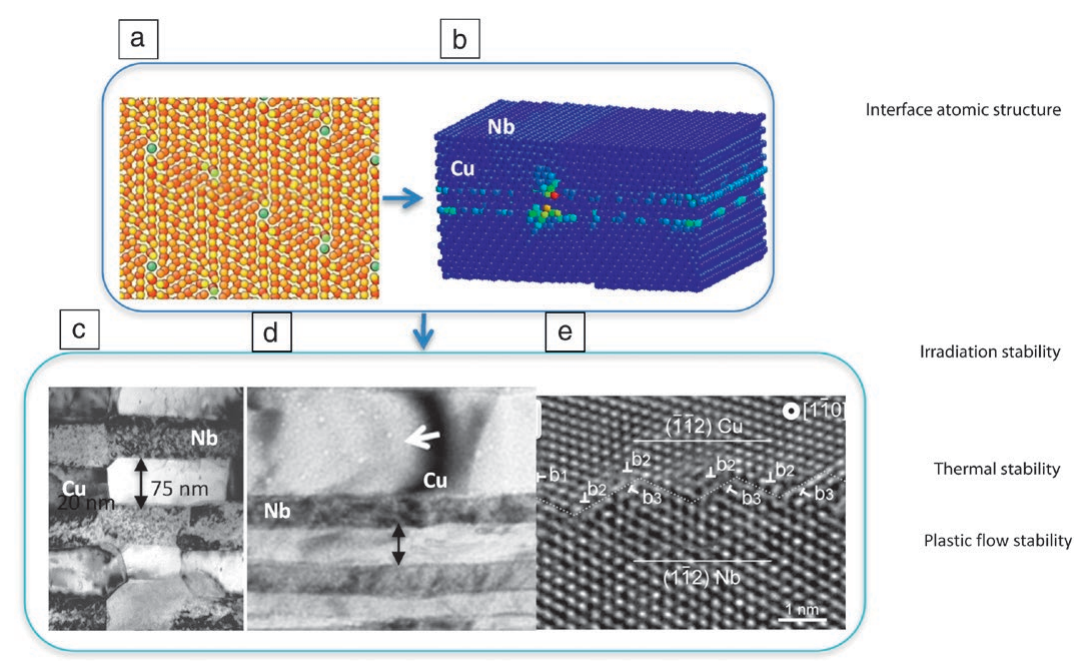

Figure 1. Example of interface-enabled behavior in model $\mathrm{Cu}-\mathrm{Nb}$ metallic nanocomposites. (a) Interface atomic structures that lead to low shear strength interfaces that (b) shear under the local stress field of an impinging glide dislocation that gets trapped via core spreading in the interface plane. In addition to high yield strength, these interfaces give rise to unprecedented morphological stability at elevated temperatures (c), under ion irradiation (d), and under high plastic strains during severe plastic deformation processing (e). in confined systems where the crystal symmetry breaks down at nanometer-spaced interfaces. The interplay between dislocation slip and deformation twinning in the noncubic side of the interface and the interface-dominant behavior at the nanoscale imply that new models are needed to explain and predict the stress-strain response and texture evolution. Integration of models at different length scales that incorporate the physics of the unit mechanisms of dislocation and twin nucleation, blockage, storage, and recovery at interfaces to predict the anisotropic behavior of noncubic/cubic nanocomposites continues to be a challenge and the focus of many ongoing investigations, as discussed by Beyerlein and Wang in their article in this issue..$^{20}$

Given the large fraction of interface area per unit volume in nanocomposites, interfaces enable the evolution of defects, such as defect (dislocation or deformation twin) nucleation, blockage, absorption (storage), and annihilation (recovery), which determines the overall
ehavior - strength, strain hardening, ductility, mechanical behavior - strength, strain hardening, ductility,
toughness, deformation textures, and morphological stabilityunder extremes of irradiation, temperature, and high strain rates. ${ }^{12}$

Using a series of immiscible, noncompound-forming $\mathrm{Cu}-\mathrm{X}$ model systems, where $\mathrm{X}$ is a bcc metal $(\mathrm{Nb}, \mathrm{V}, \mathrm{Cr}, \mathrm{Mo}, \mathrm{W}$, $\mathrm{Ta}$, and $\mathrm{Fe}$ ), it has been shown that semicoherent interfaces exhibit unique atomic structures involving multiple atomic arrangements with low and nearly degenerate interface energies. ${ }^{5,14}$ As a result of this unique atomic-scale design, significant challenge. Recent work on morphological design of metallic nanocomposites, discussed in the Demkowicz article in this issue, ${ }^{18}$ shows that the shape of the interfaces, in conjunction with the interface spacing, is critical in designing materials to suppress shear bands at ultrahigh yield strengths. ${ }^{18,19}$

The exploration of noncubic/cubic composites (e.g., lamellar $\mathrm{Al}_{2} \mathrm{Cu}-\mathrm{Al}$; hexagonal close-packed-Mg/body-centered cubic $[\mathrm{bcc}]-\mathrm{Nb}$ ) has provided insights into the plasticity mechanisms

Table I. Summary of interface-enabled mechanical behavior in metallic nanocomposites comprised of immiscible elements and semicoherent interfaces.

Property of Designed Interface

Interfaces "weak" in shear

High interfacial barrier for dislocation transmission

Interfaces with low interfacial energies and multiple atomic arrangements with nearly degenerate energies

Interfaces with location-dependent energies for interstitials

Ordered atomic-scale interfacial defect structure to influence deformation twin or glide dislocation nucleation barriers

Bicontinuous, intertwined interfaces that are "weak" in shear

Hierarchical morphologies with hard/soft nanophases and geometrically aligned slip systems

\section{Critical Unit Mechanism}

Glide dislocation trapping in interface plane due to core delocalization

Confined layer, symmetric slip in nanolaminates $=>$ high strain hardening uniform distribution of slip

Low formation energy of point defects at interfaces.

Core delocalization of point defects.

Low driving force for interface migration.

Nanoscale confinement of helium precipitates at misfit dislocation intersections

Interfaces that favor or suppress deformation twinning

Suppression of shear localization

Interfaces that favor slip transmission in disparate phases

\section{Consequence on Mechanical Behavior}

High yield strength ${ }^{1,22}$

High plastic flow and crystallographic stability; ${ }^{25,35}$ High fatigue strength; ${ }^{26}$ wear resistance ${ }^{27}$

Thermal and irradiation stability, high-temperature strength $22,24,28-31$

Helium management and outgassing in structural materials for nuclear energy ${ }^{32,33}$

Deformation mechanisms that control (1) shock response ${ }^{34}$ and (2) deformation textures in severe plastic deformation processing ${ }^{25,35}$

High yield strength and ductility ${ }^{19}$

Plastic co-deformability in high-strength metal-intermetallic ${ }^{36}$ and metal-metallic glass systems ${ }^{9-11}$ 
interfaces in $\mathrm{Cu}-\mathrm{Nb}$ and related systems exhibit unusual behavior (e.g., very high cohesive strength but relatively low shear strength in the interface plane), which leads to localized shear in response to impinging glide dislocations and trapping of dislocations in the interface plane with delocalized (spread) $\operatorname{core}^{5}$ (Figure 1a-b). This also results in a very high interface barrier to slip transmission that can be tailored via design of the interface atomic structure to influence the interface shear strength. ${ }^{21}$ The flow strength of such nanocomposites for normal loading or parallel to interfaces is extremely high. ${ }^{1,22}$ The core delocalization of line as well as point defects in the interface plane combined with low interface energies and multiple atomic arrangements in the interface with degenerate energies is the fundamental basis for maintaining the morphological and crystallographic stability of interfaces. $\mathrm{Cu}-\mathrm{Nb}$ metallic nanocomposites were shown to be stable after annealing at temperatures up to $800^{\circ} \mathrm{C}$, Figure $1 \mathrm{c},{ }^{23}$ after helium ion irradiation at $400^{\circ} \mathrm{C}$ where radiation-induced voids formed in the bulk $\mathrm{Cu}$, but not in the nanolaminates, Figure $1 \mathrm{~d},{ }^{24}$ and after high plastic strains during severe plastic deformation processing via accumulative roll bonding, Figure 1e. ${ }^{25}$

Several examples of interface-enabled behavior for metallic nanocomposites are summarized in Table I. Recent works have also explored fatigue and wear resistance, ${ }^{26,27}$ and high-temperature strength and radiation behavior. ${ }^{28-33}$ The defect structure of interphase boundaries at the atomic scale has been shown to correlate with nucleation of glide dislocations and deformation twinning that is relevant to controlling the "twinnability" of interfaces under shock ${ }^{34}$ and severe plastic deformation. ${ }^{25,35}$ Finally, the morphology of the interfaces in nanocomposites has been shown to be effective in controlling plastic deformability - bicontinuous, intertwined nanocomposites seem to suppress shear localization better than nanolaminates, ${ }^{36}$ and hierarchical morphologies can lead to plastic co-deformability in nanocomposites with soft/ hard phases. ${ }^{36}$

\section{Nanocarbon, soft, and bioinspired nanocomposites}

The integration of nanocarbons such as graphene and nanotubes with metals to synthesize nanocomposites has resulted in new materials with ultrahigh strengths, toughness, and electrical conductivity values. ${ }^{37-39}$ Graphenereinforced (2.5 vol\%) Cu matrix composites with a nacre-inspired nanolaminated architecture and improved interfacial bonding were shown to increase yield strength by approximately $177 \%$ and elastic modulus by approximately $25 \%$ compared to the unreinforced $\mathrm{Cu}$ matrix, while maintaining the ductility and electrical conductivity of the copper. ${ }^{38}$

In metal/graphene/metal nanocomposites, the graphene sheets provide a new way to engineer metallic grain boundaries to provide increased strength by blocking dislocations as well as hindering crack propagation, leading to toughening and fatigue resistance, as described in article by Guo et al. in this issue. ${ }^{40}$ Graphene-reinforced polymer-matrix nanocomposites have been extensively studied and are reviewed elsewhere. ${ }^{41}$ The challenge of alignment of graphene sheets was addressed by a stacking and folding method to generate aligned graphene/polycarbonate composites ${ }^{42}$ up to 0.11 $\mathrm{mm}$ in thickness with increased effective elastic modulus and strength, and retention of anisotropic electrical conduction along the graphene planar axis.

Nacre-inspired Al-graphene nanocomposites with interlocked nanolaminated architectures were reported to exhibit enhancement in hardness, tensile strength, and fatigue life; these were synthesized via a multistep powder-metallurgy/ extrusion/laser shock peening route. ${ }^{43}$ The production of a
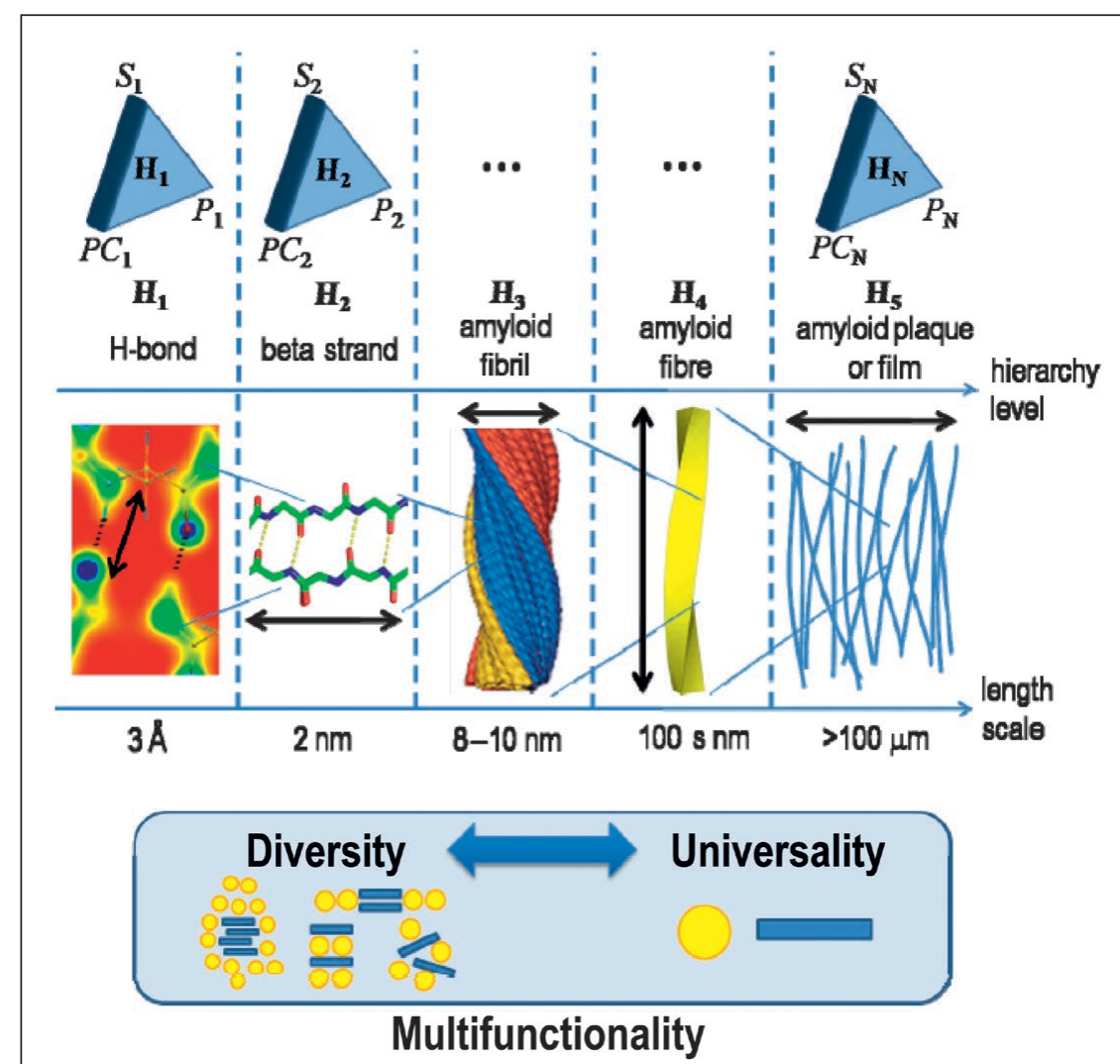

Figure 2. Creating functional diversity out of the systematic and directed assembly of material constituents at defined length scales. This nested, hierarchical approach allows one to engineer structure and properties at each scale by defining a specific process, leading to interactive and tunable material designs. $P C_{N}$ is the process at level $N ; S_{N}$ is the structure at level $N$; and $P_{N}$ is the property at level $N$. Hydrogen bonds $\left(\mathrm{H}_{1}\right)$ with characteristic lengths on the order of a fraction of a nanometer provide the chemical bonding that forms beta strands $\left(\mathrm{H}_{2}\right)$, which in turn, form highly ordered, twisted fibrils $\left(\mathrm{H}_{3}\right)$. The fibrils grow into long fibers $\left(\mathrm{H}_{4}\right)$ that are then arranged into plaques or films through a casting process $\left(\mathrm{H}_{5}\right)$. Reprinted with permission from Reference 46. (๐) 2010 Springer Nature. 
lamellar morphology, control of interface "weakness" for sliding through an interlock mechanism, and confinement of dislocation loops to interlamellar regions ${ }^{43}$ were all shown to be crucial for producing the observed enhancements in mechanical properties.

Soft materials represent an active area of materials investigations in important contexts such as robotics, biomaterials, and sensors. Recent advances allow for the integrated computational, theoretical, and manufacturing of complex materials by design. Through the articles by Ren et al. ${ }^{44}$ and Pro and Barthelat ${ }^{45}$ in this issue, we highlight a selection of advances in this area, focused especially on the combination of structure and dissimilar material constituents in composites to achieve new properties.

The paradigm of interface-enabled mechanical behavior is also emerging in the mechanical behavior of soft and biomaterials-based nanocomposites. ${ }^{44}$ For example, in silk/ carbon nanocomposites, as described in an article by Ren et al. in their article, 44 "weak" interfaces induce nonlinear deformations and deflect cracks making propagation more difficult, and gradient interfaces channel programmed deformation of materials to enable environment-adaptive behavior. The combination of silk and hard materials, especially new generations of carbon materials, offers exciting prospects to achieve superior multifunctional properties. Such new material designs are particularly noteworthy because they combine material classes that are not usually integrated, either in nature or in engineering.
Silk is one of the strongest and toughest, as well as the most versatile protein-based biofiber (dominated by weak H-bonds), while carbon materials represent a class of synthetically engineered building blocks for some of the strongest materials known (dominated by covalent bonds). Engineering of the interfaces between these disparate materials offers a rich class of design solutions and new interaction phenomena that drive larger-scale material functions. Of particular interest in this area is the interplay of strong and weak bonds.

Likewise, the phenomenon of nanoscale ductility caused by weak interfaces is found to be ubiquitous in biological composites, as described in the Pro and Barthelat article. ${ }^{45}$ However, efforts to mimic such materials such as nacre in artificial systems has the limitation that statistical variations and imperfections in the material architecture lead to strain localization and brittle fracture, pointing to the challenge of duplicating nature's amazing synthesis capacity in lab-synthesized materials. Novel strategies involving 3D printed, large-scale bioinspired materials are presented that have shown significant promise.

\section{Conclusion}

The field of composites has seen several major developments in recent years, especially in our ability to design and synthesize novel materials, and using the directed design of structure across multiple length scales, as depicted schematically in Figure 2. ${ }^{46}$ As we expand the design space across length and time scales,
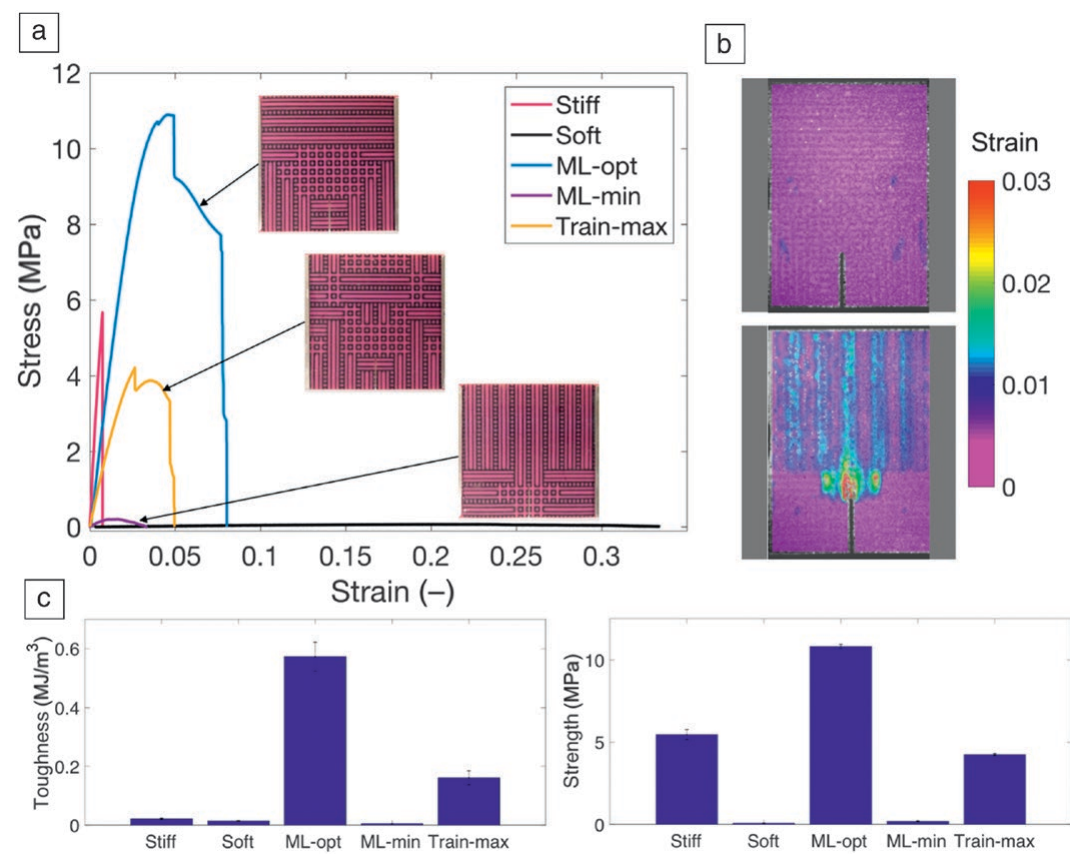

Figure 3. Additive manufacturing and subsequent testing of hierarchical composites designed using machine learning (ML). (a) Comparison of stress-strain response of ML-generated 3D printed sample (ML-opt) to its (soft and stiff) building blocks, lowest toughness geometry obtained from machine learning (ML-min), and the maximum toughness geometry from the training set (train-max). Three-dimensional printed designs for ML-opt, ML-min, and train-max are shown as insets in the figure. (b) Strain field plots obtained from digital image correlation for ML-opt (top) and ML-min (bottom). (c) Toughness and strength values for the various designs. ${ }^{48}$ we are now able to tune properties simultaneously. One of the early examples is the combination of strength and toughness, but the concept also offers the integration of mechanical, optical, electrical, and other properties. Further, with the ability to change structures at distinct scales, material properties can be tuned after a material is fabricated, for uses as actuators, sensors, or to adapt material properties. Such types of design objectives rely increasingly on structural complexity, which requires the need for new design tools to aid the parameter search in a vast space of possible solutions. An important aspect is the process by which materials are made, which becomes part of the design solution.

The use of novel modeling techniques, such as machine learning, combined with additive manufacturing, opens new avenues for future research that complements physics-based methods with data-driven approaches such as machine learning (Figure 3). ${ }^{47,48}$ Machine learning offers the opportunity to capture the physics of a particular design space of interest in a neural network, trained by data derived from experiments or first-principles multiscale modeling, facilitating a rapid inquiry of performance measures for variations in the design. The key issue here is that neural networks perform much faster than typical multiscale methods and hence allow 
for rapid improvements of designs, which can be iterated using genetic algorithms or gradient-based methods. ${ }^{47,48}$ Moreover, these methods can be designed as autonomous algorithms, where the neural network can be improved by adding more data to its training set by spawning new first-principles-based multiscale modeling, if more accuracy is needed.

Another exciting opportunity that directly intersects with computing is in recent advances in additive manufacturing (Figure 3) and other novel methods. These can lead to numerous insights and practical applications in the context of materials by design. Laser-based processing, such as direct metal deposition and other related approaches, has significant potential in the synthesis of designed metallic nanocomposites of the future. ${ }^{49,50}$ Likewise, 3D printing ${ }^{51}$ has shown promise through high spatial fidelity, flexibility, and high throughput to process large-scale bioinspired architectured materials with improved mechanical properties.

An interesting concept is the integration of neural networkbased algorithms with additive manufacturing, offering a path to allow autonomous networks to actually produce a physical artefact and then test it, and return the data back to the model for improvement and enhancing the predictive capability. This type of setup may involve the use of advanced robotics to perform material testing for the purpose of manufacturing and processing and material characterization. An autonomous system like this may be able to rapidly evolve material design, processing, and characterization in a truly autonomous manner without human intervention. The opportunities for composite design, but also the development of new polymers, alloys, and other material classes, are endless. ${ }^{52}$

\section{Acknowledgments}

A.M. acknowledges support from the US Department of Energy, Basic Energy Sciences, on plasticity in laser-processed nanoscale eutectics (DE-SC0016808), and NSF-DMREF on design of PVD-synthesized bicontinuous, intertwined nanocomposites (1533557). M.J.B. acknowledges support from the US Department of Defense, Office of Naval Research (N0001416-1-233), the National Institutes of Health (U01 EB014976), and DOD-MURI (FA9550-15-1-0514).

\section{References}

1. N.A. Mara, D. Bhattacharyya, P. Dickerson, R.G. Hoagland, A. Misra, Appl. Phys. Lett. 92, 231901 (2008).

2. Y. Li, D. Raabe, M. Herbig, P.-P. Choi, S. Goto, A. Kostka, H. Yarita, C. Borchers, R. Kirchheim, Phys. Rev. Lett. 113, 106104 (2014).

3. L.-B. Mao, H.-L. Gao, H.-B. Yao, L. Liu, H. Cölfen, G. Liu, S.-M. Chen, S.-K. Li, Y.-X. Yan, Y.-Y. Liu, S.-H. Yu, Science 354, 107 (2016).

4. M. Koyama, Z. Zhang, M. Wang, D. Ponge, D. Raabe, K. Tsuzaki, H. Noguchi, C.C. Tasan, Science 355, 1055 (2017).

5. R.G. Hoagland, J.P. Hirth, A. Misra, Philos. Mag. 86, 3537 (2006)

6. P.M. Anderson, J.F. Bingert, A. Misra, J.P. Hirth, Acta Mater. 51, 6059 (2003).

7. I. Salehinia, S. Shao, J. Wang, H.M. Zbib, Acta Mater. 86, 331 (2015).

8. L.W. Yang, C. Mayer, N. Li, J.K. Baldwin, N.A. Mara, N. Chawla, J.M. MolinaAldareguia, J. Llorca, Acta Mater. 142, 37 (2018).

9. B. Cheng, J.R. Trelewicz, Acta Mater. 153, 314 (2018).

10. Y. Wang, D. Kiener, X. Liang, J. Bian, K. Wu, J. Zhang, G. Liu, J. Sun, J. Alloys Compd. 768, 88 (2018).

11. I. Knorr, N.M. Cordero, E.T. Lilleodden, C.A. Volkert, Acta Mater. 61, 4984 (2013). 12. I.J. Beyerlein, M.J. Demkowicz, A. Misra, B.P. Uberuaga, Prog. Mater. Sci. 74, 125 (2015).
13. J.P. Hirth, R.C. Pond, R.G. Hoagland, X.-Y. Liu, J. Wang, Prog. Mater. Sci. 58, 749 (2013).

14. M.J. Demkowicz, J. Wang, R.G. Hoagland, Dislocations in Solids: A Tribute to F.R.N. Nabarro, North-Holland Publishing, Amsterdam, The Netherlands, (2008), vol. 14, pp. 141-205.

15. J. Wang, Q. Zhou, S. Shao, A. Misra, Mater. Res. Lett. 5 (1), 1 (2017).

16. N.A. Mara, I.J. Beyerlein, Curr. Opin. Solid State Mater. Sci. 19, 265 (2015).

17. S.M. Han, M.A. Phillips, W.D. Nix, Acta Mater. 57, 4473 (2009).

18. M.J. Demkowicz, MRS Bull. 44 (1), 25 (2019).

19. Y. Cui, B. Derby, N. Li, N.A. Mara, A. Misra, Mater. Res. Lett. 6, 184 (2018)

20. I.J. Beyerlein, J. Wang, MRS Bull. 44 (1), 31 (2019).

21. J. Wang, A. Misra, R.G. Hoagland, J.P. Hirth, Acta Mater. 60, 1503 (2012).

22. T. Nizolek, I.J. Beyerlein, N.A. Mara, J.T. Avallone, T.M. Pollock, Appl. Phys. Lett. 108, 051903 (2016)

23. A. Misra, R.G. Hoagland, H. Kung, Philos. Mag. 84, 1021 (2004).

24. W. Han, M.J. Demkowicz, N.A. Mara, E.G. Fu, S. Sinha, A.D. Rollett, Y.Q. Wang, J.S. Carpenter, I.J. Beyerlein, A. Misra, Adv. Mater. 25, 6975 (2013).

25. I.J. Beyerlein, J.R. Mayeur, J. Wang, S.J. Zheng, N.A. Mara, A. Misra, Proc. Natl. Acad. Sci. U.S.A. 111, 4386 (2014).

26. Y.C. Wang, A. Misra, R.G. Hoagland, Scr. Mater. 54, 1593 (2006).

27. R. Madhavan, P. Bellon, R.S. Averback, ACS Appl. Mater. Interfaces 10, 15288 (2018)

28. M.A. Monclus, S.J. Zheng, J. Mayeur, I.J. Beyerlein, N.A. Mara, T. Polcar, J. Llorca, J. Molina-Aldareguia, APL Mater. 1, 052103 (2013).

29. S.J. Dillon, D.C. Bufford, G.S. Jawaharram, K. Hattar, X. Liu, C. Lear, R.S. Averback, J. Nucl. Mater. 490, 59 (2017).

30. X. Zhang, S. Shu, P. Bellon, R.S. Averback, Acta Mater. 97, 348 (2015).

31. X. Zhang, K. Hattar, Y. Chen, L. Shao, J. Li, C. Sun, K. Yu, N. Li, M. L. Taheri, H. Wang, J. Wang, M. Nastasi, Prog. Mater. Sci. 96, 217 (2018).

32. A. Kashinath, M.J. Demkowicz, A. Misra, Phys. Rev. Lett. 110, 086101 (2013).

33. D. Chen, N. Li, D. Yuryev, J.K. Baldwin, Y.Q. Wang, M.J. Demkowicz, Sci. Adv. 3, eaa02710, (2017).

34. W.Z. Han, E.K. Cerreta, N.A. Mara, I.J. Beyerlein, J.S. Carpenter, S.J. Zheng, C.P. Trujillo, P.O. Dickerson, A. Misra, Acta Mater. 63, 150 (2014).

35. A. Misra, R.G. Hoagland, J. Mater. Sci. 42, 1765 (2007).

36. S.J. Wang, G. Liu, D.Y. Xie, Q. Lei, B.P. Ramakrishnan, J. Mazumder, J. Wang, A. Misra, Acta Mater. 156, 52 (2018).

37. M. Yang, L. Weng, H.X. Zhu, T.X. Fan, D. Zhang, Carbon 118, 250 (2017).

38. M. Cao, D.-B. Xiong, Z.Q. Tan, G. Ji, B. Amin-Ahmadi, Q. Guo, G.L. Fan, C.P. Guo, Z.Q. Li, D. Zhang, Carbon 117, 65 (2017).

39. A. Nieto, A. Bisht, D. Lahiri, C. Zhang, A. Agarwal, Int. Mater. Rev. 62 (5), 241 (2017).

40. Q. Guo, K. Kondoh, S.M. Han, MRS Bull. 44 (1), 40 (2019).

41. D.G. Papageorgiou, I.A. Kinloch, R.J. Young, Prog. Mater. Sci. 90,75 (2017).

42. P.W. Liu, Z. Jin, G. Katsukis, L.W. Drahushuk, S. Shimizu, C.J. Shih, E.D. Wetzel, J.K. Taggart-Scarff, B. Qing, K.J. Van Vliet, R. Li, B.L. Wardle, M.S. Strano, Science 353, 364 (2016).

43. H.G.P. Kumar, S. Prabhakaran, M.A. Xavior, S. Kalainathan, D. Lin, P. Shukla, V.K. Vasudevan, Mater. Today Commun. 16, 81 (2018).

44. J. Ren, Y. Liu, D.L. Kaplan, S. Ling, MRS Bull. 44 (1), 53 (2019).

45. J.W. Pro, F. Barthelat, MRS Bull. 44 (1), 46 (2019).

46. M.J. Buehler, Nat. Nanotechnol. 5, 172 (2010).

47. G.X. Gu, C.-T. Chen, M.J. Buehler, Extreme Mech. Lett. 18, 19 (2018).

48. G.X. Gu, C.T. Chen, D.J. Richmond, M.J. Buehler, Mater. Horiz. 5 (5), 939 (2018).

49. Y. Huang, S.R. Schmid, J. Manuf. Sci. Eng. 137, 094001 (2015).

50. B.P. Ramakrishnan, Q. Lei, J. Mazumder, A. Misra, Sci. Rep. 7, 13468 (2017). 51. L.S. Dimas, G.H. Bratzel, I. Eylon, M.J. Buehler, Adv. Funct. Mater. 23, 4629 (2013).

52. I.A. Kinloch, J. Suhr, J. Lou, R.J. Young, P.M. Ajayan, Science 362, 547 (2018).

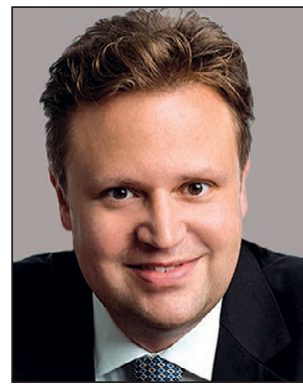

Markus J. Buehler is a McAfee Professor of Engineering at the Massachusetts Institute of Technology (MIT). His research focuses on new modeling, design, and manufacturing approaches for advanced materials that offer greater resilience and controllable properties from the nano- to the macroscale. He has numerous publications, including his latest book, Biomateriomics. His awards include the MRS Outstanding Young Investigator Award, the MIT Harold E. Edgerton Faculty Achievement Award, the Alfred Noble Prize of the American Society of Civil Engineers, and the Leonardo da Vinci Award. Buehler can be reached by email at mbuehler@mit.edu. 


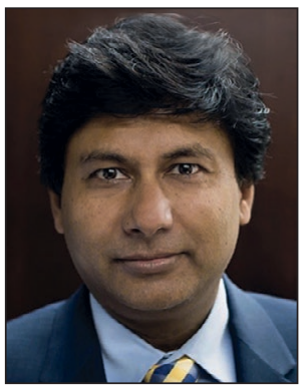

Amit Misra has been a professor and chair of the Department of Materials Science and Engineering at the University of Michigan since June 2014. From 1996 to 2014, he was employed at Los Alamos National Laboratory (LANL). His research group studies nanomechanics and metallic interfaces focusing on the design and development of multiphase and nanocomposite materials for advanced structural applications. He is a Fellow of MRS, ASM International, and LANL. Misra can be reached by email at amitmis@umich.edu.
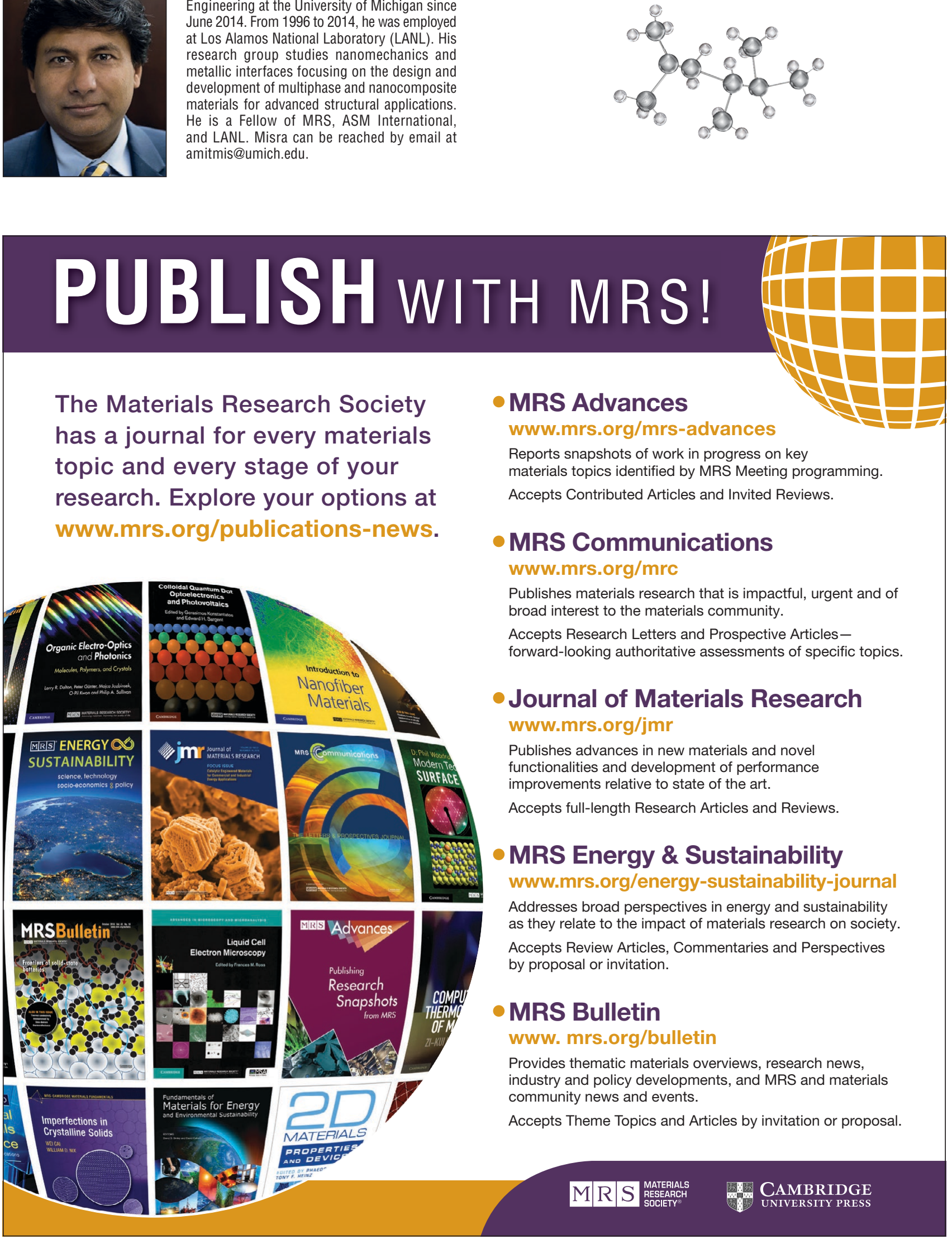